\title{
ASAP: Adaptive Structure Aware Pooling for Learning Hierarchical Graph Representations
}

\author{
Ekagra Ranjan, ${ }^{1 *}$ Soumya Sanyal, ${ }^{2}$ Partha Talukdar ${ }^{2}$ \\ ${ }^{1}$ Indian Institute of Technology, Guwahati \\ ${ }^{2}$ Indian Institute of Science, Bangalore \\ ekagra.ranjan@gmail.com, \{soumyasanyal,ppt\}@iisc.ac.in
}

\begin{abstract}
Graph Neural Networks (GNN) have been shown to work effectively for modeling graph structured data to solve tasks such as node classification, link prediction and graph classification. There has been some recent progress in defining the notion of pooling in graphs whereby the model tries to generate a graph level representation by downsampling and summarizing the information present in the nodes. Existing pooling methods either fail to effectively capture the graph substructure or do not easily scale to large graphs. In this work, we propose ASAP (Adaptive Structure Aware Pooling), a sparse and differentiable pooling method that addresses the limitations of previous graph pooling architectures. ASAP utilizes a novel self-attention network along with a modified GNN formulation to capture the importance of each node in a given graph. It also learns a sparse soft cluster assignment for nodes at each layer to effectively pool the subgraphs to form the pooled graph. Through extensive experiments on multiple datasets and theoretical analysis, we motivate our choice of the components used in ASAP. Our experimental results show that combining existing GNN architectures with ASAP leads to state-of-the-art results on multiple graph classification benchmarks. ASAP has an average improvement of $4 \%$, compared to current sparse hierarchical state-of-the-art method. We make the source code of ASAP available to encourage reproducible research ${ }^{1}$.
\end{abstract}

\section{Introduction}

In recent years, there has been an increasing interest in developing Graph Neural Networks (GNNs) for graph structured data. CNNs have shown to be successful in tasks involving images (Krizhevsky, Sutskever, and Hinton 2012; He et al. 2016) and text (Kim 2014). Unlike these regular grid data, arbitrary shaped graphs have rich information present in their graph structure. By inherently capturing such information through message propagation along the edges of the graph, GNNs have proved to be more effective for graphs (Gilmer et al. 2017; Hamilton, Ying,

\footnotetext{
${ }^{*}$ Research done during internship at Indian Institute of Science, Bangalore.

Copyright (C) 2020, Association for the Advancement of Artificial Intelligence (www.aaai.org). All rights reserved.

${ }^{1}$ https://github.com/malllabiisc/ASAP
}

and Leskovec 2017). While some of the works focus on learning node-level representations to perform tasks such as node classification (Kipf and Welling 2017; Veličković et al. 2017) and link prediction (Schlichtkrull et al. 2017; Vashishth et al. 2019), others focus on learning graph-level representations for tasks like graph classification (Bruna et al. 2013; Henaff, Bruna, and LeCun 2015; Ying et al. 2018; Gao and Ji 2019; Lee, Lee, and Kang 2019). In this paper, we focus on graph-level representation learning for the task of graph classification.

Briefly, the task of graph classification involves predicting the label of an input graph by utilizing the given graph structure and initial node-level representations. For example, given a molecule, the task could be to predict if it is toxic. Current GNNs are inherently flat and lack the capability of aggregating node information in a hierarchical manner. Such architectures rely on learning node representations through some GNN followed by aggregation of the node information to generate the graph representation (Vinyals, Bengio, and Kudlur 2016; Li et al. 2016; Zhang et al. 2018). But learning graph representations in a hierarchical manner is important to capture local substructures that are present in graphs. For example, in an organic molecule, a set of atoms together can act as a functional group and play a vital role in determining the class of the graph.

To address this limitation, new pooling architectures have been proposed where sets of nodes are recursively aggregated to form a cluster that represents a node in the pooled graph, thus enabling hierarchical learning. DiffPool (Ying et al. 2018) is a differentiable pooling operator that learns a soft assignment matrix mapping each node to a set of clusters. Since this assignment matrix is dense, it is not easily scalable to large graphs (Cangea et al. 2018). Following that, TopK (Gao and Ji 2019) is proposed which learns a scalar projection score for each node and selects the top $k$ nodes. They address the sparsity concerns of DiffPool but are unable to capture the rich graph structure effectively. Recently, SAGPool (Lee, Lee, and Kang 2019), a TopK based architecture, has been proposed which leverages self-attention network to learn the node scores. Although local graph structure is used for scoring nodes, it is still not used effectively in determin- 


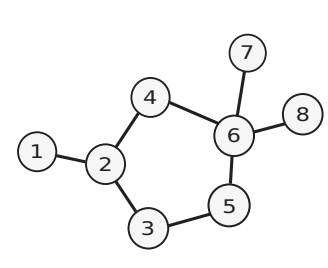

(a) Input graph

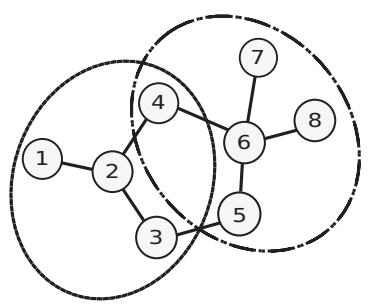

(b) Cluster assignment and formation

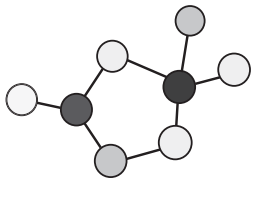

(c) Clusters scoring using LEConv
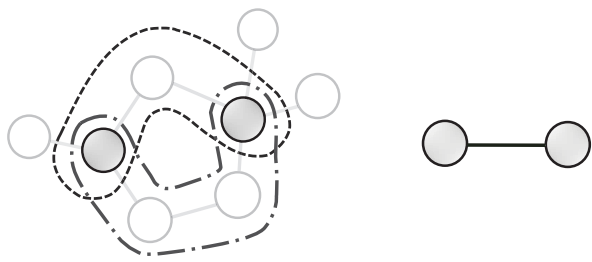

(d) Top scoring clusters are selected (e) Pooled graph

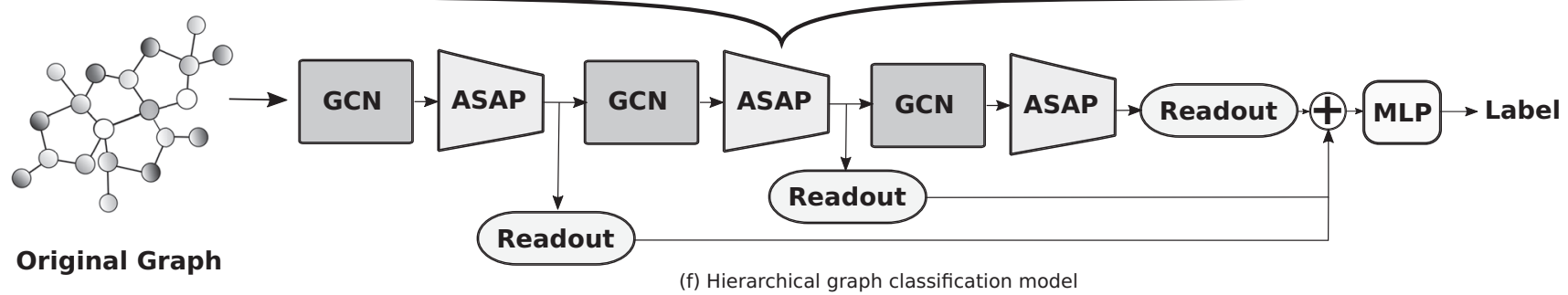

Figure 1: Overview of ASAP: (a) Input graph to ASAP. (b) ASAP initially clusters 1-hop neighborhood considering all nodes as medoid ${ }^{2}$. For brevity, we only show cluster formations of nodes $2 \& 6$ as medoids. Cluster membership is computed using M2T attention (refer Sec. 4.2). (c) Clusters are scored using LEConv (refer Sec. 4.3). Darker shade denotes higher score. (d) A fraction of top scoring clusters are selected in the pooled graph. Adjacency matrix is recomputed using edge weights between the member nodes of selected clusters. (e) Output of ASAP (f) Overview of hierarchical graph classification architecture.

ing the connectivity of the pooled graph. Pooling methods that leverage the graph structure effectively while maintaining sparsity currently don't exist. We address the gap in this paper.

In this work, we propose a new sparse pooling operator called Adaptive Structure Aware Pooling (ASAP) which overcomes the limitations in current pooling methods. Our contributions can be summarized as follows:

- We introduce ASAP, a sparse pooling operator capable of capturing local subgraph information hierarchically to learn global features with better edge connectivity in the pooled graph.

- We propose Master2Token (M2T), a new self-attention framework which is better suited for global tasks like pooling.

- We introduce a new convolution operator LEConv, that can adaptively learn functions of local extremas in a graph substrucutre.

\section{Related Work}

\subsection{Graph Neural Networks}

Various formulation of GNNs have been proposed which use both spectral and non-spectral approaches. Spectral methods (Bruna et al. 2013; Henaff, Bruna, and LeCun 2015) aim at defining convolution operation using Fourier transformation and graph Laplacian. These methods do not directly generalize to graphs with different structure (Bronstein et al.

\footnotetext{
${ }^{2}$ medoids are representatives of a cluster. They are similar to centroids but are strictly a member of the cluster.
}

2017). Non-spectral methods (Defferrard, Bresson, and Vandergheynst 2016; Kipf and Welling 2017; Xu et al. 2018; Monti et al. 2017; Morris et al. 2018) define convolution through a local neighborhood around nodes in the graph. They are faster than spectral methods and easily generalize to other graphs. GNNs can also be viewed as message passing algorithm where nodes iteratively aggregate messages from neighboring nodes through edges (Gilmer et al. 2017).

\subsection{Pooling}

Pooling layers overcome GNN's inability to aggregate nodes hierarchically. Earlier pooling methods focused on deterministic graph clustering algorithms (Defferrard, Bresson, and Vandergheynst 2016; Fey et al. 2018; Simonovsky and Komodakis 2017). Ying et al. introduced the first differentiable pooling operator which out-performed the previous deterministic methods. Since then, new data-driven pooling methods have been proposed; both spectral (Ma et al. 2019; Dhillon, Guan, and Kulis 2007) and non-spectral (Ying et al. 2018; Gao and Ji 2019). Spectral methods aim at capturing the graph topology using eigen-decomposition algorithms. However, due to higher computational requirement for spectral graph techniques, they are not easily scalable to large graphs. Hence, we focus on non-spectral methods.

Pooling methods can further be divided into global and hierarchical pooling layers. Global pooling summarize the entire graph in just one step. Set2Set (Vinyals, Bengio, and Kudlur 2016) finds the importance of each node in the graph through iterative content-based attention. Global-Attention (Li et al. 2016) uses an attention mechanism to aggregate nodes in the graph. SortPool (Zhang et al. 2018) summarizes the graph by concatenating few nodes after sorting them based on their features. Hierarchical pooling is used to cap- 
ture the topological information of graphs. DiffPool forms a fixed number of clusters by aggregating nodes. It uses GNN to compute a dense soft assignment matrix, making it infea-

\begin{tabular}{lcccc}
\hline Property & DiffPool & TopK & SAGPool & ASAP \\
\hline Sparse & & $\checkmark$ & $\checkmark$ & $\checkmark$ \\
Node Aggregation & $\checkmark$ & & & $\checkmark$ \\
Soft Edge Weights & $\checkmark$ & & & $\checkmark$ \\
Variable number of clusters & & $\checkmark$ & $\checkmark$ & $\checkmark$ \\
\hline
\end{tabular}

Table 1: Properties desired in hierarchical pooling methods.

sible for large graphs. TopK scores nodes based on a learnable projection vector and samples a fraction of high scoring nodes. It avoids node aggregation and computing soft assignment matrix to maintain the sparsity in graph operations. SAGPool improve upon TopK by using a GNN to consider the graph structure while scoring nodes. Since TopK and SAGPool do not aggregate nodes nor compute soft edge weights, they are unable to preserve node and edge information effectively.

To address these limitations, we propose ASAP, which has all the desirable properties of hierarchical pooling without compromising on sparsity in graph operations. Please see Table. 1 for an overall comparison of hierarchical pooling methods. Further comparison discussions between hierarchical architectures are presented in Sec. 8.1.

\section{Preliminaries}

\subsection{Problem Statement}

Consider a graph $G(\mathcal{V}, \mathcal{E}, X)$ with $N=|\mathcal{V}|$ nodes and $|\mathcal{E}|$ edges. Each node $v_{i} \in \mathcal{V}$ has $d$-dimensional feature representation denoted by $x_{i} . X \in \mathbb{R}^{N \times d}$ denotes the node feature matrix and $A \in \mathbb{R}^{N \times N}$ represents the weighted adjacency matrix. The graph $G$ also has a label $y$ associated with it. Given a dataset $D=\left\{\left(G_{1}, y_{1}\right),\left(G_{2}, y_{2}\right), \ldots\right\}$, the task of graph classification is to learn a mapping $f: \mathcal{G} \rightarrow \mathcal{Y}$, where $\mathcal{G}$ is the set of input graphs and $\mathcal{Y}$ is the set of labels associated with each graph. A pooled graph is denoted by $G^{p}\left(\mathcal{V}^{p}, \mathcal{E}^{p}, X^{p}\right)$ with node embedding matrix $X^{p}$ and its adjacency matrix as $A^{p}$.

\subsection{Graph Convolution Networks}

We use Graph Convolution Network (GCN) (Kipf and Welling 2017) for extracting discriminative features for graph classification. GCN is defined as:

$$
X^{(l+1)}=\sigma\left(\hat{D}^{-\frac{1}{2}} \hat{A} \hat{D}^{\frac{1}{2}} X^{(l)} W^{(l)}\right),
$$

where $\hat{A}=A+I$ for self-loops, $\hat{D}=\sum_{j} \hat{A}_{i, j}$ and $W^{(l)} \in$ $\mathbb{R}^{d \times f}$ is a learnable matrix for any layer $l$. We use the initial node feature matrix wherever provided, i.e., $X^{(0)}=X$.

\subsection{Self-Attention}

Self-attention is used to find the dependency of an input on itself (Cheng, Dong, and Lapata 2016; Vaswani et al. 2017). An alignment score $\alpha_{i, j}$ is computed to map the importance of candidates $c_{j}$ on target query $q_{i}$. In self-attention, target query $q_{i}$ and candidates $c_{j}$ are obtained from input entities $\boldsymbol{h}=\left\{h_{1}, \ldots, h_{n}\right\}$. Self-attention can be categorized as Token2Token and Source2Token based on the choice of target query $q$ (Shen et al. 2018).

Token2Token (T2T) selects both the target and candidates from the input set $\boldsymbol{h}$. In the context of additive attention (Bahdanau, Cho, and Bengio 2014), $\alpha_{i, j}$ is computed as:

$$
\alpha_{i, j}=\operatorname{softmax}\left(\vec{v}^{T} \sigma\left(W h_{i} \| W h_{j}\right)\right) .
$$

where $\|$ is the concatenation operator.

Source2Token (S2T) finds the importance of each candidate to a specific global task which cannot be represented by any single entity. $\alpha_{i, j}$ is computed by dropping the target query term. Eq. (2) changes to the following:

$$
\alpha_{i, j}=\operatorname{softmax}\left(\vec{v}^{T} \sigma\left(W h_{j}\right)\right) .
$$

\subsection{Receptive Field}

We extend the concept of receptive field $R F$ from pooling operations in $\mathrm{CNN}$ to $\mathrm{GNN}^{3}$. We define $R F^{\text {node }}$ of a pooling operator as the number of hops needed to cover all the nodes in the neighborhood that influence the representation of a particular output node. Similarly, $R F^{e d g e}$ of a pooling operator is defined as the number of hops needed to cover all the edges in the neighborhood that affect the representation of an edge in the pooled graph $\mathcal{G}^{p}$.

\section{ASAP: Proposed Method}

In this section we describe the components of our proposed method ASAP. As shown in Fig. 1(b), ASAP initially considers all possible local clusters with a fixed receptive field for a given input graph. It then computes the cluster membership of the nodes using an attention mechanism. These clusters are then scored using a GNN as depicted in Fig 1(c). Further, a fraction of the top scoring clusters are selected as nodes in the pooled graph and new edge weights are computed between neighboring clusters as shown in Fig. 1(d). Below, we discuss the working of ASAP in details. Please refer to Appendix Sec. I for a pseudo code of the working of ASAP.

\subsection{Cluster Assignment}

Initially, we consider each node $v_{i}$ in the graph as a medoid of a cluster $c_{h}\left(v_{i}\right)$ such that each cluster can represent only the local neighbors $\mathcal{N}$ within a fixed radius of $h$ hops i.e., $c_{h}\left(v_{i}\right)=\mathcal{N}_{h}\left(v_{i}\right)$. This effectively means that $R F^{n o d e}=h$ for ASAP. This helps the clusters to effectively capture the information present in the graph sub-structure.

Let $x_{i}^{c}$ be the feature representation of a cluster $c_{h}\left(v_{i}\right)$ centered at $v_{i}$. We define $G^{c}\left(\mathcal{V}, \mathcal{E}, X^{c}\right)$ as the graph with node feature matrix $X^{c} \in \mathbb{R}^{N \times d}$ and adjacency matrix $A^{c}=A$. We denote the cluster assignment matrix by $S \in \mathbb{R}^{N \times N}$, where $S_{i, j}$ represents the membership of node $v_{i} \in \mathcal{V}$ in

${ }^{3}$ Please refer to Appendix Sec. D for more details on similarity between pooling methods in $\mathrm{CNN}$ and ASAP. 
cluster $c_{h}\left(v_{j}\right)$. By employing such local clustering (Schaeffer 2007), we can maintain sparsity of the cluster assignment matrix $S$ similar to the original graph adjacency matrix $A$ i.e., space complexity of both $S$ and $A$ is $\mathcal{O}(|\mathcal{E}|)$.

\subsection{Cluster Formation using Master2Token}

Given a cluster $c_{h}\left(v_{i}\right)$, we learn the cluster assignment matrix $S$ through a self-attention mechanism. The task here is to learn the overall representation of the cluster $c_{h}\left(v_{i}\right)$ by attending to the relevant nodes in it. We observe that both T2T and S2T attention mechanisms described in Sec. 3.3 do not utilize any intra-cluster information. Hence, we propose a new variant of self-attention called Master2Token (M2T). We further motivate the need for M2T framework later in Sec. 8.2. In M2T framework, we first create a master query $m_{i} \in \mathbb{R}^{d}$ which is representative of all the nodes within a cluster:

$$
\left.m_{i}=f_{m}\left(x_{j}^{\prime} \mid v_{j} \in c_{h}\left(v_{i}\right)\right\}\right),
$$

where $x_{j}^{\prime}$ is obtained after passing $x_{j}$ through a separate GCN to capture structural information in the cluster $c_{h}\left(v_{i}\right)^{4}$. $f_{m}$ is a master function which combines and transforms feature representation of $v_{j} \in c_{h}\left(v_{i}\right)$ to find $m_{i}$. In this work we experiment with $\max$ master function defined as:

$$
m_{i}=\max _{v_{j} \in c_{h}\left(v_{i}\right)}\left(x_{j}^{\prime}\right) .
$$

This master query $m_{i}$ attends to all the constituent nodes $v_{j} \in c_{h}\left(v_{i}\right)$ using additive attention:

$$
\alpha_{i, j}=\operatorname{softmax}\left(\vec{w}^{T} \sigma\left(W m_{i} \| x_{j}^{\prime}\right)\right) .
$$

where $\vec{w}^{T}$ and $W$ are learnable vector and matrix respectively. The calculated attention scores $\alpha_{i, j}$ signifies the membership strength of node $v_{j}$ in cluster $c_{h}\left(v_{i}\right)$. Hence, we use this score to define the cluster assignment matrix discussed above, i.e., $S_{i, j}=\alpha_{i, j}$. The cluster representation $x_{i}^{c}$ for $c_{h}\left(v_{i}\right)$ is computed as follows:

$$
x_{i}^{c}=\sum_{j=1}^{\left|c_{h}\left(v_{i}\right)\right|} \alpha_{i, j} x_{j} .
$$

\subsection{Cluster Selection using LEConv}

Similar to TopK (Gao and Ji 2019), we sample clusters based on a cluster fitness score $\phi_{i}$ calculated for each cluster in the graph $G^{c}$ using a fitness function $f_{\phi}$. For a given pooling ratio $k \in(0,1]$, the top $\lceil k N\rceil$ clusters are selected and included in the pooled graph $G^{p}$. To compute the fitness scores, we introduce Local Extrema Convolution (LEConv), a graph convolution method which can capture local extremum information. In Sec. 5.1 we motivate the choice of LEConv's formulation and contrast it with the standard GCN formulation. LEConv is used to compute $\phi$ as follows:

$$
\phi_{i}=\sigma\left(x_{i}^{c} W_{1}+\sum_{j \in \mathcal{N}(i)} A_{i, j}^{c}\left(x_{i}^{c} W_{2}-x_{j}^{c} W_{3}\right)\right)
$$

${ }^{4}$ If $x_{j}$ is used as it is then interchanging any two nodes in a cluster will have not affect the final output, which is undesirable. where $\mathcal{N}(i)$ denotes the neighborhood of the $i^{t h}$ node in $G^{c}$. $W_{1}, W_{2}, W_{3}$ are learnable parameters and $\sigma($.$) is some acti-$ vation function. Fitness vector $\Phi=\left[\phi_{1}, \phi_{2}, \ldots, \phi_{N}\right]^{T}$ is multiplied to the cluster feature matrix $X^{c}$ to make $f_{\phi}$ learnable i.e.,:

$$
\hat{X}^{c}=\Phi \odot X^{c},
$$

where $\odot$ is broadcasted hadamard product. The function $\operatorname{TOP}_{k}($.$) ranks the fitness scores and gives the indices \hat{i}$ of top $\lceil k N\rceil$ selected clusters in $G^{c}$ as follows:

$$
\hat{i}=\operatorname{TOP}_{k}\left(\hat{X}^{c},\lceil k N\rceil\right) \text {. }
$$

The pooled graph $G^{p}$ is formed by selecting these top $\lceil k N\rceil$ clusters. The pruned cluster assignment matrix $\hat{S} \in$ $\mathbb{R}^{N \times\lceil k N\rceil}$ and the node feature matrix $X^{p} \in \mathbb{R}^{\lceil k N\rceil \times d}$ are given by:

$$
\hat{S}=S(:, \hat{i}), X^{p}=\hat{X}^{c}(\hat{i},:)
$$

where $\hat{i}$ is used for index slicing.

\subsection{Maintaining Graph Connectivity}

Following (Ying et al. 2018), once the clusters have been sampled, we find the new adjacency matrix $A^{p}$ for the pooled graph $G^{p}$ using $\hat{A}^{c}$ and $\hat{S}$ in the following manner:

$$
A^{p}=\hat{S}^{T} \hat{A}^{c} \hat{S}
$$

where $\hat{A}^{c}=A^{c}+I$. Equivalently, we can see that $A_{i, j}^{p}=$ $\sum_{k, l} \hat{S}_{k, i} \hat{A}_{k, l}^{c} \hat{S}_{l, j}$. This formulation ensures that any two clusters $i$ and $j$ in $G^{p}$ are connected if there is any common node in the clusters $c_{h}\left(v_{i}\right)$ and $c_{h}\left(v_{j}\right)$ or if any of the constituent nodes in the clusters are neighbors in the original graph $G$ (Fig. 1(d)). Hence, the strength of the connection between clusters is determined by both the membership of the constituent nodes through $\hat{S}$ and the edge weights $A^{c}$. Note that $\hat{S}$ is a sparse matrix by formulation and hence the above operation can be implemented efficiently.

\section{Theoretical Analysis \\ 5.1 Limitations of using GCN for scoring clusters}

GCNs cannot learn to assign such a fitness score to a cluster which is a function of the local extremas of its constituent nodes. Scoring the clusters based on local extremas would potentially allow us to sample representative clusters from all parts of the graph. GCN from Eq. (1) can be viewed as an operator which first computes a pre-score $\hat{\phi}^{\prime}$ for each node i.e., $\hat{\phi}^{\prime}=X W$ followed by a weighted average over neighbors and a non-linearity. If for some node the pre-score is very high, it can increase the scores of its neighbors, inherently biasing the pooling operators to select a node in the local neighborhood instead of sampling clusters which represent the whole graph.

Theorem 1. Let $\mathcal{G}$ be a graph with positive adjacency matrix $A$ i.e., $A_{i, j} \geq 0$. Consider any function $f(X, A)$ : $\mathbb{R}^{N \times d} \times \mathbb{R}^{N \times N} \rightarrow \mathbb{R}^{N \times 1}$ which depends on difference between a node and its neighbors after a linear transformation $W \in \mathbb{R}^{d \times 1}$. For e.g,:

$$
f_{i}=\sigma\left(\alpha_{i} x_{i} W+\sum_{j \in \mathcal{N}(i)} \beta_{i, j}\left(x_{i} W-x_{j} W\right)\right)
$$




\begin{tabular}{lccccc}
\hline Method & D\&D & PROTEINS & NCI1 & NCI109 & FRANKENSTEIN \\
\hline SET2SET (Vinyals, Bengio, and Kudlur 2016) & $71.60 \pm 0.87$ & $72.16 \pm 0.43$ & $66.97 \pm 0.74$ & $61.04 \pm 2.69$ & $61.46 \pm 0.47$ \\
GLOBAL-ATTENTION (Li et al. 2016) & $71.38 \pm 0.78$ & $71.87 \pm 0.60$ & $69.00 \pm 0.49$ & $67.87 \pm 0.40$ & $61.31 \pm 0.41$ \\
SoRTPoOL (Zhang et al. 2018) & $71.87 \pm 0.96$ & $73.91 \pm 0.72$ & $68.74 \pm 1.07$ & $68.59 \pm 0.67$ & $63.44 \pm 0.65$ \\
\hline DifFPOOL (Ying et al. 2018) & $66.95 \pm 2.41$ & $68.20 \pm 2.02$ & $62.32 \pm 1.90$ & $61.98 \pm 1.98$ & $60.60 \pm 1.62$ \\
TOPK (Gao and Ji 2019) & $75.01 \pm 0.86$ & $71.10 \pm 0.90$ & $67.02 \pm 2.25$ & $66.12 \pm 1.60$ & $61.46 \pm 0.84$ \\
SAGPOOL (Lee, Lee, and Kang 2019) & $76.45 \pm 0.97$ & $71.86 \pm 0.97$ & $67.45 \pm 1.11$ & $67.86 \pm 1.41$ & $61.73 \pm 0.76$ \\
\hline ASAP (Ours) & $\mathbf{7 6 . 8 7} \pm \mathbf{0 . 7}$ & $\mathbf{7 4 . 1 9} \pm \mathbf{0 . 7 9}$ & $\mathbf{7 1 . 4 8} \pm \mathbf{0 . 4 2}$ & $\mathbf{7 0 . 0 7} \pm \mathbf{0 . 5 5}$ & $\mathbf{6 6 . 2 6} \pm \mathbf{0 . 4 7}$ \\
\hline
\end{tabular}

Table 2: Comparison of ASAP with previous global and hierarchical pooling. Average accuracy and standard deviation is reported for 20 random seeds. We observe that ASAP consistently outperforms all the baselines on all the datasets. Please refer to Sec. 7.1 for more details.

where $f_{i}, \alpha_{i}, \beta_{i, j} \in \mathbb{R}$ and $x_{i} \in \mathbb{R}^{d}$.

a) If fitness value $\Phi=G C N(X, A)$ then $\Phi$ cannot learn $f$.

b) If fitness value $\Phi=L E \operatorname{Conv}(X, A)$ then $\Phi$ can learn $f$.

Proof. See Appendix Sec. F for proof.

Motivated by the above analysis, we propose to use LEConv (Eq. 8) for scoring clusters. LEConv can learn to score clusters by considering both its global and local importance through the use of self-loops and ability to learn functions of local extremas.

\subsection{Graph Connectivity}

Here, we analyze ASAP from the aspect of edge connectivity in the pooled graph. When considering $h$-hop neighborhood for clustering, both ASAP and DiffPool have $R F^{e d g e}=2 h+1$ because they use Eq. (10) to define the edge connectivity. On the other hand, both TopK and SAGPool have $R F^{e d g e}=h$. A larger edge receptive field implies that the pooled graph has better connectivity which is important for the flow of information in the subsequent GCN layers.

Theorem 2. Let the input graph $\mathcal{G}$ be a tree of any possible structure with $N$ nodes. Let $k^{*}$ be the lower bound on sampling ratio $k$ to ensure the existence of atleast one edge in the pooled graph irrespective of the structure of $\mathcal{G}$ and the location of the selected nodes. For TopK or SAGPool, $k^{*} \rightarrow 1$ whereas for ASAP, $k^{*} \rightarrow 0.5$ as $N \rightarrow \infty$.

Proof. See Appendix Sec. G for proof.

Theorem 2 suggests that ASAP can achieve a similar degree of connectivity as SAGPool or TopK for a much smaller sampling ratio $k$. For a tree with no prior information about its structure, ASAP would need to sample only half of the clusters whereas TopK and SAGPool would need to sample almost all the nodes, making TopK and SAGPool inefficient for such graphs. In general, independent of any combination of nodes selected, ASAP will have better connectivity due to its larger receptive field. Please refer to Appendix Sec. G for a similar analysis on path graph and more details.

\subsection{Graph Permutation Equivariance}

Proposition 1. ASAP is a graph permutation equivariant pooling operator.

Proof. See Appendix Sec. H for proof.

\section{Experimental Setup}

In our experiments, we use 5 graph classification benchmarks and compare ASAP with multiple pooling methods. Below, we describe the statistics of the dataset, the baselines used for comparisons and our evaluation setup in detail.

\subsection{Datasets}

We demonstrate the effectiveness of our approach on 5 graph classification datasets. D\&D (Shervashidze et al. 2011; Dobson and Doig 2003) and PROTEINS (Dobson and Doig 2003; Borgwardt et al. 2005) are datasets containing proteins as graphs. NCI1 (Wale, Watson, and Karypis 2008) and NCI109 are datasets for anticancer activity classification. FRANKENSTEIN (Orsini, Frasconi, and De Raedt 2015) contains molecules as graph for mutagen classification. Please refer to Table 3 for the dataset statistics.

\begin{tabular}{lcccc}
\hline Dataset & $\mathrm{G}_{\text {avg }}$ & $\mathrm{C}_{\text {avg }}$ & $\mathrm{V}_{\text {avg }}$ & $\mathrm{E}_{\text {avg }}$ \\
\hline D\&D & 1178 & 2 & 284.32 & 715.66 \\
PROTEINS & 1113 & 2 & 39.06 & 72.82 \\
NCI1 & 4110 & 2 & 29.87 & 32.30 \\
NCI109 & 4127 & 2 & 29.68 & 32.13 \\
FRANKENSTEIN & 4337 & 2 & 16.90 & 17.88 \\
\hline
\end{tabular}

Table 3: Statistics of the graph datasets. $\mathrm{G}_{a v g}, \mathrm{C}_{a v g}, \mathrm{~V}_{a v g}$ and $\mathrm{E}_{\text {avg }}$ denotes the average number of graphs, classes, nodes and edges respectively.

\subsection{Baselines}

We compare ASAP with previous state-of-the-art hierarchical pooling operators DiffPool (Ying et al. 2018), TopK (Gao and Ji 2019) and SAGPool (Lee, Lee, and Kang 2019). For comparison with global pooling, we choose Set2Set (Vinyals, Bengio, and Kudlur 2016), Global-Attention (Li et al. 2016) and SortPool (Zhang et al. 2018). 


\subsection{Training \& Evaluation Setup}

We use a similar architecture as defined in (Cangea et al. 2018; Lee, Lee, and Kang 2019) which is depicted in Fig. 1(f). For ASAP, we choose $k=0.5$ and $h=1$ to be consistent with baselines. ${ }^{5}$ Following SAGPool(Lee, Lee, and Kang 2019), we conduct our experiments using 10-fold cross-validation and report the average accuracy on 20 random seeds.

\begin{tabular}{lcc}
\hline Aggregation type & FITNESS & CLUSTER \\
\hline None & - & - \\
Only cluster & - & $\checkmark$ \\
Both & $\checkmark$ & $\checkmark$ \\
\hline
\end{tabular}

Table 4: Different aggregation types as mentioned in Sec 7.2.

\section{Results}

In this section, we provide empirical analysis of ASAP by comparing it with above baselines. Next, we provide some ablation study of the various components of ASAP.

\subsection{Performance Comparison}

We compare the performace of ASAP with baseline methods on 5 graph classification tasks. The results are shown in Table 2. All the numbers for hierarchical pooling (DiffPool, TopK and SAGPool) are taken from (Lee, Lee, and Kang 2019). For global pooling (Set2Set, Global-Attention and SortPool), we modify the architectural setup to make them comparable with the hierarchical variants. ${ }^{6}$. We observe that ASAP consistently outperforms all the baselines on all 5 datasets. We note that ASAP has an average improvement of $4 \%$ and $3.5 \%$ over previous state-of-the-art hierarchical (SAGPool) and global (SortPool) pooling methods respectively. We also observe that compared to other hierarchical methods, ASAP has a smaller variance in performance which suggests that the training of ASAP is more stable.

\subsection{Effect of Node Aggregation}

Here, we evaluate the improvement in performance due to our proposed technique of aggregating nodes to form a cluster. There are two aspects involved during the creation of clusters for a pooled graph:

- FITNESS: calculating fitness scores for individual nodes. Scores can be calculated either by using only the medoid or by aggregating neighborhood information.

- CLUSTER: generating a representation for the new cluster node. Cluster representation can either be the medoid's representation or some feature aggregation of the neighborhood around the medoid.

\footnotetext{
${ }^{5}$ Please refer to Appendix Sec. A for further details on hyperparameter tuning and Appendix Sec. $\mathrm{E}$ for ablation on $k$.

${ }^{6}$ Please refer to Appendix Sec. B for more details
}

We test three types of aggregation methods: 'None', 'Only cluster' and 'Both' as described in Table 4. As shown in Table 5 , we observe that our proposed node aggregation helps improve the performance of ASAP.

\begin{tabular}{lcc}
\hline Aggregation & FRANKENSTEIN & NCI1 \\
\hline None & $67.4 \pm 0.6$ & $69.9 \pm 2.5$ \\
Only cluster & $67.5 \pm 0.5$ & $70.6 \pm 1.8$ \\
Both & $\mathbf{6 7 . 8} \pm \mathbf{0 . 6}$ & $\mathbf{7 0 . 7} \pm \mathbf{2 . 3}$ \\
\hline
\end{tabular}

Table 5: Performace comparison of different aggregation methods on validation data of FRANKENSTEIN and NCI1.

\begin{tabular}{lcc}
\hline Attention & FRANKENSTEIN & NCI1 \\
\hline T2T & $67.6 \pm 0.5$ & $70.3 \pm 2.0$ \\
S2T & $67.7 \pm 0.5$ & $69.9 \pm 2.0$ \\
M2T & $\mathbf{6 7 . 8} \pm \mathbf{0 . 6}$ & $\mathbf{7 0 . 7} \pm \mathbf{2 . 3}$ \\
\hline
\end{tabular}

Table 6: Effect of different attention framework on pooling evaluated on validation data of FRANKENSTEIN and NCI1. Please refer to Sec. 7.3 for more details.

\subsection{Effect of M2T Attention}

We compare our M2T attention framework with previously proposed $\mathrm{S} 2 \mathrm{~T}$ and $\mathrm{T} 2 \mathrm{~T}$ attention techniques. The results are shown in Table 6. We find that M2T attention is indeed better than the rest in NCI1 and comparable in FRANKENSTEIN.

\begin{tabular}{lcc}
\hline Fitness function & FRANKENSTEIN & NCI1 \\
\hline GCN & $62.7 \pm 0.3$ & $65.4 \pm 2.5$ \\
Basic-LEConv & $63.1 \pm 0.7$ & $69.8 \pm 1.9$ \\
LEConv & $\mathbf{6 7 . 8} \pm \mathbf{0 . 6}$ & $\mathbf{7 0 . 7} \pm \mathbf{2 . 3}$ \\
\hline
\end{tabular}

Table 7: Performance comparison of different fitness scoring functions on validation data of FRANKENSTEIN and NCI1. Refer to Sec. 7.4 for details.

\subsection{Effect of LEConv as a fitness scoring function}

In this section, we analyze the impact of LEConv as a fitness scoring function in ASAP. We use two baselines GCN (Eq. 1) and Basic-LEConv which computes $\phi_{i}=$ $\sigma\left(x_{i} W+\sum_{j \in \mathcal{N}\left(x_{i}\right)} A_{i, j}\left(x_{i} W-x_{j} W\right)\right)$. In Table 7 we can see that Basic-LEConv and LEConv perform significantly better than GCN because of their ability to model functions of local extremas. Further, we observe that LEConv performs better than Basic-LEConv as it has three different linear transformation compared to only one in the latter. This allows LEConv to potentially learn complicated scoring functions which is better suited for the final task. Hence, our analysis in Theorem 1 is emperically validated. 


\subsection{Effect of computing Soft edge weights}

We evaluate the importance of calculating edge weights for the pooled graph as defined in Eq. 10. We use the best model configuration as found from above ablation analysis and then add the feature of computing soft edge weights for clusters. We observe a significant drop in performace when the edge weights are not computed. This proves the necessity of capturing the edge information while pooling graphs.

\begin{tabular}{ccc}
\hline Soft edge weights & FRANKENSTEIN & NCI1 \\
\hline Absent & $67.8 \pm 0.6$ & $70.7 \pm 2.3$ \\
Present & $\mathbf{6 8 . 3} \pm \mathbf{0 . 5}$ & $\mathbf{7 3 . 4} \pm \mathbf{0 . 4}$ \\
\hline
\end{tabular}

Table 8: Effect of calculating soft edge weights on pooling for validation data of FRANKENSTEIN and NCI1. Please refer to Sec. 7.5 for more details.

\section{Discussion}

\subsection{Comparison with other pooling methods}

DiffPool DiffPool and ASAP both aggregate nodes to form a cluster. While ASAP only considers nodes which are within $h$-hop neighborhood from a node $x_{i}$ (medoid) as a cluster, DiffPool considers the entire graph. As a result, in DiffPool, two nodes that are disconnected or far away in the graph can be assigned similar clusters if the nodes and their neighbors have similar features. Since this type of cluster formation is undesirable for a pooling operator (Ying et al. 2018), DiffPool utilizes an auxiliary link prediction objective during training to specifically prevent far away nodes from being clustered together. ASAP needs no such additional regularization because it ensures the localness while clustering. DiffPool's soft cluster assignment matrix $S$ is calculated for all the nodes to all the clusters making $S$ a dense matrix. Calculating and storing this does not scale easily for large graphs. ASAP, due to the local clustering over $h$-hop neighborhood, generates a sparse assignment matrix while retaining the hierarchical clustering properties of Diffpool. Further, for each pooling layer, DiffPool has to predetermine the number of clusters it needs to pick which is fixed irrespective of the input graph size. Since ASAP selects the top $k$ fraction of nodes in current graph, it inherently takes the size of the input graph into consideration.

TopK \& SAGPool While TopK completely ignores the graph structure during pooling, SAGPool modifies the TopK formulation by incorporating the graph structure through the use of a GCN network for computing node scores $\phi$. To enforce sparsity, both TopK and SAGPool avoid computing the cluster assignment matrix $S$ that DiffPool proposed. Instead of grouping multiple nodes to form a cluster in the pooled graph, they drop nodes from the original graph based on a score (Cangea et al. 2018) which might potentially lead to loss of node and edge information. Thus, they fail to leverage the overall graph structure while creating the clusters. In contrast to TopK and SAGPool, ASAP can capture the rich graph structure while aggregating nodes to form clusters in the pooled graph. TopK and SAGPool sample edges from the original graph to define the edge connectivity in the pooled graph. Therefore, they need to sample nodes from a local neighborhood to avoid isolated nodes in the pooled graph. Maintaining graph connectivity prevents these pooling operations from sampling representative nodes from the entire graph. The pooled graph in ASAP has a better edge connectivity compared to TopK and SAGPool because soft edge weights are computed between clusters using upto three hop connections in the original graph. Also, the use of LEConv instead of GCN for finding fitness values $\phi$ further allows ASAP to sample representative clusters from local neighborhoods over the entire graph.

\subsection{Comparison of Self-Attention variants}

Source2Token \& Token2Token T2T models the membership of a node by generating a query based only on the medoid of the cluster. Graph Attention Network (GAT) (Veličković et al. 2017) is an example of T2T attention in graphs. S2T finds the importance of each node for a global task. As shown in Eq. 3, since a query vector is not used for calculating the attention scores, S2T inherently assigns the same membership score to a node for all the possible clusters that node can belong to. Hence, both S2T and T2T mechanisms fail to effectively utilize the intra-cluster information while calculating a node's cluster membership. On the other hand, M2T uses a master function $f_{m}$ to generate a query vector which depends on all the entities within the cluster and hence is a more representative formulation. To understand this, consider the following scenario. If in a given cluster, a non-medoid node is removed, then the unnormalized membership scores for the rest of the nodes will remain unaffected in S2T and T2T framework whereas the change will reflect in the scores calculated using M2T mechanism. Also, from Table 6, we find that M2T performs better than S2T and T2T attention showing that M2T is better suited for global tasks like pooling.

\section{Conclusion}

In this paper, we introduce ASAP, a sparse and differentiable pooling method for graph structured data. ASAP clusters local subgraphs hierarchically which helps it to effectively learn the rich information present in the graph structure. We propose Master2Token self-attention framework which enables our model to better capture the membership of each node in a cluster. We also propose LEConv, a novel GNN formulation that scores the clusters based on its local and global importance. ASAP leverages LEConv to compute cluster fitness scores and samples the clusters based on it. This ensures the selection of representative clusters throughout the graph. ASAP also calculates sparse edge weights for the selected clusters and is able to capture the edge connectivity information efficiently while being scalable to large graphs. We validate the effectiveness of the components of ASAP both theoretically and empirically. Through extensive experiments, we demonstrate that ASAP achieves state-ofthe-art performace on multiple graph classification datasets. 


\section{References}

Bahdanau, D.; Cho, K.; and Bengio, Y. 2014. Neural machine translation by jointly learning to align and translate. arXiv preprint arXiv:1409.0473.

Borgwardt, K. M.; Ong, C. S.; Schönauer, S.; Vishwanathan, S.; Smola, A. J.; and Kriegel, H.-P. 2005. Protein function prediction via graph kernels. Bioinformatics.

Bronstein, M. M.; Bruna, J.; LeCun, Y.; Szlam, A.; and Vandergheynst, P. 2017. Geometric deep learning: going beyond euclidean data. IEEE Signal Processing Magazine.

Bruna, J.; Zaremba, W.; Szlam, A.; and LeCun, Y. 2013. Spectral networks and locally connected networks on graphs. arXiv preprint arXiv:1312.6203.

Cangea, C.; Veličković, P.; Jovanović, N.; Kipf, T.; and Liò, P. 2018. Towards sparse hierarchical graph classifiers. arXiv preprint arXiv:1811.01287.

Cheng, J.; Dong, L.; and Lapata, M. 2016. Long short-term memory-networks for machine reading. Proceedings of the 2016 Conference on Empirical Methods in Natural Language Processing.

Defferrard, M.; Bresson, X.; and Vandergheynst, P. 2016. Convolutional neural networks on graphs with fast localized spectral filtering. In Advances in neural information processing systems.

Dhillon, I. S.; Guan, Y.; and Kulis, B. 2007. Weighted graph cuts without eigenvectors a multilevel approach. IEEE transactions on pattern analysis and machine intelligence.

Dobson, P. D., and Doig, A. J. 2003. Distinguishing enzyme structures from non-enzymes without alignments. Journal of molecular biology.

Fey, M.; Eric Lenssen, J.; Weichert, F.; and Müller, H. 2018. Splinecnn: Fast geometric deep learning with continuous b-spline kernels. In Proceedings of the IEEE Conference on Computer Vision and Pattern Recognition.

Gao, H., and Ji, S. 2019. Graph u-nets. arXiv preprint arXiv:1905.05178.

Gilmer, J.; Schoenholz, S. S.; Riley, P. F.; Vinyals, O.; and Dahl, G. E. 2017. Neural message passing for quantum chemistry. In Proceedings of the 34th International Conference on Machine Learning - Volume 70.

Hamilton, W.; Ying, Z.; and Leskovec, J. 2017. Inductive representation learning on large graphs. In Advances in Neural Information Processing Systems.

He, K.; Zhang, X.; Ren, S.; and Sun, J. 2016. Deep residual learning for image recognition. In Proceedings of the IEEE conference on computer vision and pattern recognition.

Henaff, M.; Bruna, J.; and LeCun, Y. 2015. Deep convolutional networks on graph-structured data. arXiv preprint arXiv: 1506.05163.

Kim, Y. 2014. Convolutional neural networks for sentence classification. arXiv preprint arXiv:1408.5882.

Kipf, T. N., and Welling, M. 2017. Semi-supervised classification with graph convolutional networks. In International Conference on Learning Representations (ICLR).

Krizhevsky, A.; Sutskever, I.; and Hinton, G. E. 2012. Imagenet classification with deep convolutional neural networks. In Pereira, F.; Burges, C. J. C.; Bottou, L.; and Weinberger, K. Q., eds., Advances in Neural Information Processing Systems 25.

Lee, J.; Lee, I.; and Kang, J. 2019. Self-attention graph pooling. In Proceedings of the 36th International Conference on Machine Learning.
Li, Y.; Tarlow, D.; Brockschmidt, M.; and Zemel, R. S. 2016. Gated graph sequence neural networks. CoRR abs/1511.05493.

Ma, Y.; Wang, S.; Aggarwal, C. C.; and Tang, J. 2019. Graph convolutional networks with eigenpooling. arXiv preprint arXiv:1904.13107.

Monti, F.; Boscaini, D.; Masci, J.; Rodola, E.; Svoboda, J.; and Bronstein, M. M. 2017. Geometric deep learning on graphs and manifolds using mixture model cnns. In Proceedings of the IEEE Conference on Computer Vision and Pattern Recognition.

Morris, C.; Ritzert, M.; Fey, M.; Hamilton, W. L.; Lenssen, J. E.; Rattan, G.; and Grohe, M. 2018. Weisfeiler and leman go neural: Higher-order graph neural networks.

Orsini, F.; Frasconi, P.; and De Raedt, L. 2015. Graph invariant kernels. In Twenty-Fourth International Joint Conference on Artificial Intelligence.

Schaeffer, S. E. 2007. Graph clustering. Computer science review. Schlichtkrull, M.; Kipf, T. N.; Bloem, P.; Berg, R. v. d.; Titov, I.; and Welling, M. 2017. Modeling relational data with graph convolutional networks. arXiv preprint arXiv:1703.06103.

Shen, T.; Zhou, T.; Long, G.; Jiang, J.; Pan, S.; and Zhang, C. 2018. Disan: Directional self-attention network for $\mathrm{rnn} / \mathrm{cnn}$-free language understanding. In Thirty-Second AAAI Conference on Artificial Intelligence.

Shervashidze, N.; Schweitzer, P.; Leeuwen, E. J. v.; Mehlhorn, K.; and Borgwardt, K. M. 2011. Weisfeiler-lehman graph kernels. Journal of Machine Learning Research.

Simonovsky, M., and Komodakis, N. 2017. Dynamic edgeconditioned filters in convolutional neural networks on graphs. In Proceedings of the IEEE conference on computer vision and pattern recognition.

Vashishth, S.; Sanyal, S.; Nitin, V.; and Talukdar, P. 2019. Composition-based multi-relational graph convolutional networks. arXiv preprint arXiv:1911.03082.

Vaswani, A.; Shazeer, N.; Parmar, N.; Uszkoreit, J.; Jones, L.; Gomez, A. N.; Kaiser, Ł.; and Polosukhin, I. 2017. Attention is all you need. In Advances in neural information processing systems.

Veličković, P.; Cucurull, G.; Casanova, A.; Romero, A.; Lio, P.; and Bengio, Y. 2017. Graph attention networks. arXiv preprint arXiv:1710.10903.

Vinyals, O.; Bengio, S.; and Kudlur, M. 2016. Order matters: Sequence to sequence for sets. In International Conference on Learning Representations (ICLR).

Wale, N.; Watson, I. A.; and Karypis, G. 2008. Comparison of descriptor spaces for chemical compound retrieval and classification. Knowledge and Information Systems.

Xu, K.; Hu, W.; Leskovec, J.; and Jegelka, S. 2018. How powerful are graph neural networks?

Ying, R.; You, J.; Morris, C.; Ren, X.; Hamilton, W. L.; and Leskovec, J. 2018. Hierarchical graph representation learning with differentiable pooling. In Proceedings of the 32Nd International Conference on Neural Information Processing Systems.

Zhang, M.; Cui, Z.; Neumann, M.; and Chen, Y. 2018. An end-toend deep learning architecture for graph classification. In ThirtySecond AAAI Conference on Artificial Intelligence. 